\title{
Developmentally Regulated Alternative Splicing Generates a Complex Array of Drosophila para Sodium Channel Isoforms
}

\author{
Justin R. Thackeray and Barry Ganetzky \\ Laboratory of Genetics, University of Wisconsin, Madison, Wisconsin 53706
}

\begin{abstract}
The para locus encodes the predominant class of sodium channels expressed in Drosophila neurons. Previous sequence analysis of para CDNAs indicated the occurrence of alternative splicing at several sites within the open reading frame. Here we report a detailed analysis of this alternative splicing and its regulation during development. We have used a combination of RNA-PCR and sequence analysis to examine a 1.7 kilobase region of the para mRNA that encompasses the previously reported sites of alternative splicing. Five sites of alternative splicing were identified; 48 different splice variants could be generated by the differential exon usage observed. The number of splice forms and their relative frequency in vivo were characterized in RNA samples of both embryos and adults. The range of splice types was found to be much more diverse in adults than in embryos; of a total of 19 different combinations of alternative exons, 11 splice types were found in embryos and 18 in adults. Usage of some individual alternative exons changed during development; a newly identified exon, which is found in one of two forms either 24 or $\mathbf{3 0}$ base pairs long, was present in about $85 \%$ of para transcripts from embryos but only $7 \%$ of those in adults. These data suggest that a wide variety of subtly distinct $\mathrm{Na}$ channel isoforms are present in Drosophila, and that these may provide a range of voltagegated sodium channel functions. Although multiple sodium channel genes have already been described in both Drosophila and mammalian systems, this study provides a clear indication that sodium channel variability may be much greater than previously thought.
\end{abstract}

[Key words: Drosophila, neurogenetics, para, sodium channel, alternative splicing, RNA-PCR]

Voltage-sensitive sodium ( $\mathrm{Na}$ ) channels play a key role in electrical signaling in the nervous system. Forming a conduction pathway for sodium ions across the neuronal membrane, they activate in response to membrane depolarization and mediate the propagation of action potentials along the axon (Hodgkin and Huxley, 1952). Following their initial biochemical purifi-

\footnotetext{
Received June 29, 1993; revised Oct. 1, 1993; accepted Oct. 14, 1993.

We thank Mark Tanouye for providing the para cDNA P15 and Bill Engels for statistical advice and software. We are also indebted to past and present members of the Ganetzky laboratory for both technical advice and comments on the manuscript. This work was supported by a NATO postdoctoral fellowship from the Science and Engineering Research Council (UK) to J.R.T. and by grants from the Markey Charitable Trust and National Institutes of Health (GM43100) and a Klingeristein fellowship to B.G. This is paper 3370 from the Laboratory of Genetics, University of Wisconsin, Madison.

Correspondence should be addressed to Dr. Barry Ganetzky, Laboratory of Genctics, University of Wisconsin, 445 Henry Mall, Madison, WI 53706.

Copyright (C) 1994 Society for Neuroscience 0270-6474/94/142569-10\$05.00/0
}

cation from the electroplax of electric eel (Noda et al., 1984), $\mathrm{Na}$ channels have been the subject of intensive study and much has been learned of their subunit composition, primary structure, gating properties, and voltage-sensing apparatus (for a recent review, see Hille, 1992).

Mutations of the Drosophila paralytic (para) gene cause temperature-sensitive paralysis of larvae and adults (Suzuki et al., 1971) by eliminating the conduction of axonal action potentials at elevated temperatures (Siddiqi and Benzer, 1976; Wu and Ganetzky, 1980). These phenotypes were interpreted as being the result of a sodium channel defect. Confirmation that para encodes the $\alpha$-subunit of an Na channel was provided by cloning and sequence analysis of the gene (Loughney et al., 1989; Ramaswami et al., 1989). This showed that the predicted para polypeptide has the four homology domain structure seen in all other voltage-gated $\mathrm{Na}$ channels (e.g., Guy and Conti, 1990; Hille, 1992) and a high level of amino acid sequence identity with other known $\mathrm{Na}$ channel proteins. Sequence comparison of multiple para cDNAs indicated that at least three different forms of the para protein are generated by alternative splicing (Loughney et al., 1989), by the optional inclusion of three exons, a, b, and e (encoding 21, 8, and 13 amino acids, respectively), and the choice between two further exons, $c$ and $d$, which arc of the same length but different sequence.

The importance of alternative splicing in generating functional diversity of a wide range of proteins is being increasingly recognized, not least among genes expressed in the nervous system. For example, alternative splicing of the Drosophila Shaker transcript generates at least seven voltage-sensitive potassium channel proteins, each with different electrophysiological properties when expressed in Xenopus oocytes (reviewed by Perney and Kaczmarek, 1991); the different splice forms are also differentially distributed in the nervous system (Schwarz et al., 1990). Previous work in rat has indicated that alternative splicing may also contribute to the functional diversity of $\mathrm{Na}$ channels (Sarao et al., 1991; Yarowsky et al., 1991; Schaller et al., 1992). In this article, we examine alternative splicing of the para transcript to assess its importance to $\mathrm{Na}$ channel function in Drosophila.

The sequence and position of at least two para alternative exons suggest that they may be of functional importance. First, the exon a sequence contains potential sites for phosphorylation by both cAMP-dependent protein kinase and protein kinase $\mathrm{C}$ (PKC). Li et al. (1992) have shown that phosphorylation by a cAMP-dependent protein kinase reduces the peak $\mathrm{Na}^{+}$current of a rat (RIIA) sodium channel by $40-50 \%$ both in vitro and within intact neurons. The likely site of this phosphorylation is within the first cytoplasmic linking domain (Rossie et al., 1987; Rossie and Catterall, 1989), which is where para exon a resides. 


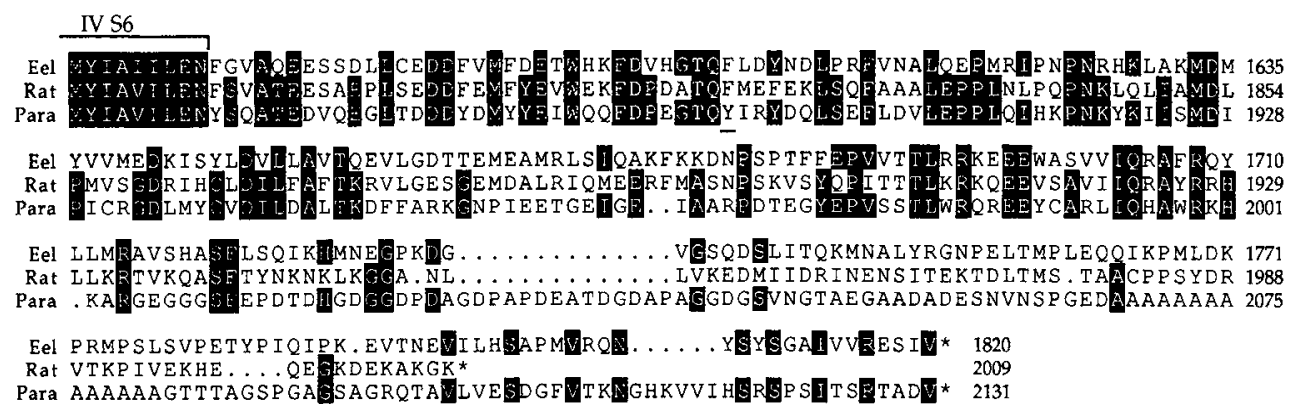

Figure 1. Alignment of sodium channel protein sequences from the carboxy-terminal region. The protein sequence at the carboxy terminus of para, deduced from cDNA P15, is shown in an alignment with the equivalent region from the rat (I) (Noda et al., 1986) and eel (Noda et al., 1984) sodium channel proteins, using the one-letter code for amino acids. Residues that are identical to the para sequence are highlighted; an asterisk marks the end of each protein. The underlined tyrosine $(Y)$ at position 1896 of the para sequence marks the first residue of the new sequence determined here. Note that the final two amino acids given by Loughney et al. (1989) were actually part of the EcoRI linker used in the cDNA library construction; the correct sequence (as also given by Ramaswami and Tanouye, 1989) is shown here. The alignment was generated by the multiple sequence alignment program PILEUP from version 7.0 of the UWGCG package (Devereux et al., 1984).

Only one other potential site exists for phosphorylation by cAMPdependent protein kinase in this region of the para protein. The potential PKC phosphorylation site may also be significant, because it has been shown that phosphorylation by PKC can lead to both a reduction in $\mathrm{Na}^{+}$current and altered voltage dependence (Sigel and Baur, 1988; Dascal and Lotan, 1991). In addition, Schaller et al. (1992) recently identified a site in three rat $\mathrm{Na}$ channel genes that can be spliced in one of six different ways. This site lies within the cytoplasmic linking region between homology domains I and II, close to the site of para exon a, and some of these alternative exons also contain a potential site for PKC phosphorylation.

The other alternative splice site that might have functional importance is within homology domain II, where either exon c or exon $\mathrm{d}$ is present. One of the two amino acid differences between $\mathrm{c}$ and $\mathrm{d}$ is at the end of the $\mathrm{S} 5$ domain, where charged residues may be partially exposed to the lumen of the channel (Guy and Conti, 1990). Because this amino acid difference involves a charged residue (threonine in exon $\mathrm{c}$ is replaced by histidine in exon d), it is possible that it contributes to the charged motif within S4 that forms the voltage sensor, and thereby affects the voltage dependence of gating. The other amino acid difference between exons $c$ and $d$, although a rather conscrvative exchange of methionine for valinc, lics only seven residues away from the site of a mutation in a skeletal muscle $\mathrm{Na}$ channel that causes hyperkalemic periodic paralysis in humans (Ptácek et al., 1991). The mutation, which also involves a methionine residue (changed from threonine in the wild-type $\mathrm{Na}$ channel), has recently been shown to cause aberrant channel inactivation (Cannon and Strittmatter, 1993).

We report here an extensive analysis of a subregion of the para transcript that encompasses the region known to be alternatively spliced. Sixteen new splice types were present among the many partial para cDNAs recovered from both embryos and adults, including a new alternative exon that is preferentially expressed in embryos. This represents the largest number of different $\mathrm{Na}$ channel isoforms isolated in any system to date. The range of different splice types produced alters dramatically during development, being much narrower in embryos than adults. These data add to a growing body of evidence that, far from being relatively uniform, $\mathrm{Na}$ channels (and presumably $\mathrm{Na}^{+}$currents) form a diverse class of protein, with many subtly distinct isoforms generated by alternative splicing.

\section{Materials and Methods}

General recombinant DNA methods. Plasmid DNA minipreparation, restriction endonuclease digests, agarose and polyacrylamide gel electrophoresis, Southern blotting, radiolabeling of DNA probes, and filter hybridization were all performed using standard methodology (Sambrook et al., 1989). Northern blotting was as described by Drysdale et al. (1991); the blot was kindly provided by Dr. J. Warmke (University of Wisconsin, Madison).

PCR amplification and cloning of para transcripts. RNA was prepared from unstaged (0-24 hr) Canton-S embryos or whole adult flies by the phenol/chloroform $/ \mathrm{LiCl}$ precipitation method described by Sambrook et al. (1989). cDNA was synthesized from $20 \mu \mathrm{g}$ of total RNA, in the presence of $1 \mu \mathrm{M}$ random hexamers (Pharmacia, Piscataway, NJ) as primers, $100 \mathrm{~mm}$ dithiothreitol (DTI), 5 mm each dNTP, $5 \mathrm{U}$ of RNasin ribonuclease inhibitor (Promega, Madison, WI), and $100 \mathrm{U}$ of murine leukemia virus reverse transcriptase in the buffer supplied by the manufacturer (GIBCO-Bethesda Research Labs, Grand Island, NY), for 1 $\mathrm{hr}$ at $37^{\circ} \mathrm{C}$. para transcripts were amplified from $2 \mu \mathrm{l}$ of cDNA in a 50 $\mu l$ reaction containing $50 \mathrm{pmol}$ of $5^{\prime}$ and $3^{\prime}$ primers $5 \mathrm{R} 2 \mathrm{~L}$, and $3 \mathrm{R} 2 \mathrm{I}$ (ACACCAAGCTTCAAAGTTCGT and CTCCTGGATCCCCTTCTTGATG, respectively), $200 \mu \mathrm{M}$ each dNTP, and $5 \mathrm{U}$ of Taq polymerase (Promega) in the buffer supplied by the manufacturer. The underlined $\mathrm{T}$ in 5R2L and $\mathrm{G}$ in $3 \mathrm{R} 2 \mathrm{~L}$ are not present in the para sequence; they were used to create a restriction site in each primer (HindIII and BamHI in $5 \mathrm{R} 2 \mathrm{~L}$ and $3 \mathrm{R} 2 \mathrm{~L}$, respectively) for cloning purposes. The amplification was performed in a Coy Tempcycler for 20 cycles of 1 $\min 94^{\circ} \mathrm{C}, 1 \min 55^{\circ} \mathrm{C}, 2 \min 72^{\circ} \mathrm{C}$, followed by 20 further cycles with a longer extension time of $4 \mathrm{~min}$ at $72^{\circ} \mathrm{C}$ to maximize product yield. The broad band of product at $\sim 1.7$ kilobases $(\mathrm{kb})$ was recovered from a $0.6 \%$ agarose gel by electrophoresis onto dialysis membrane (Sambrook et al., 1989). After ethanol precipitation, the DNA was digested with restriction enzymes BamHI and HindIII, ligated into BamHI/ HindIII-digested pBluescript KS + (Stratagene, La Jolla, CA), and used to transform Escherichia coli strain XL-I Blue (Stratagene) by electroporation. Plasmid DNA was prepared from many recombinant clones by alkaline lysis (Sambrook et al., 1989). Single-stranded DNA was prepared by polyethylene glycol precipitation (Sambrook et al., 1989) and dideoxy sequencing performed with ${ }^{35} S$-labeled dATP in combination with the Sequenase 2.0 sequencing kit (U.S. Biochemicals, Cleveland, $\mathrm{OH}$ ) and several gene-specific primers. Sequencing reactions were separated on $0.4-\mathrm{mm}$-thick $5.5 \%$ Long Ranger acrylamide (J. T. Baker, Phillipsburg, NJ), $8 \mathrm{~m}$ urea gels.

RNase protection assay. The RNase protection assay was performed with an RPA II kit (Ambion, Austin, TX), following the protocol provided by the manufacturer. The probes used all crossed at least one exon/intron boundary so that any contaminating Drosophila genomic DNA would produce protected fragments smaller than any predicted from para mRNA. Each of the four probes contained a single alternative exon constructed as follows: the exon a probe was a 395 base pair (bp) HindIII/FspI fragment (equivalent to positions 1903-2237 in cDNA ZS10.3; Loughney et al., 1989), from one of the para PCR-derived 
cDNAs described in this study, subcloned into HindIII/EcoRV-digested pBluescript KS+; the exon b probe was a $588 \mathrm{bp} \mathrm{HincII/ClaI} \mathrm{fragment}$ from cDNA ZS10.3 (1999-2587 in ZS10.3) subcloned into EcoRV/ClaIdigested pBluescript SK+; the exon c probe was a $495 \mathrm{bp} \mathrm{Xhol/StuI}$ fragment (2903-3398 in ZS10.3) subcloned into Xhol/EcoRV-digested pBluescript KS+; the exon e probe was a 415 bp PstI/BamHI fragment (3199-3575 in ZS10.3) generated by PCR with primer PS 1 (TGCTCTGCAGAGAATGGATC) and 3R2L (described above) using the cDNA ZS20.2 (Loughney et al., 1989) as template, creating a PstI site (underlined) at position 3199, subcloned into PstI/BamHI-digested pBluescript KS+. Minipreparations of plasmid DNA were linearized with a restriction enzyme (exon a probe with XhoI; b with BamHI; c with XhoI; e first with Pst I, the 3' overhang then removed with Klenow polymerase), treated with RNase A to remove residual RNA, extracted once with phenol/chloroform/isoamyl alcohol $(25: 24: 1)$ and once with chloroform, precipitated with ethanol, and resuspended in water.

Antisense RNA probes were synthesized in vitro using $0.5-1.0 \mu \mathrm{g}$ of DNA template in a $20 \mu \mathrm{l}$ reaction containing $50 \mu \mathrm{Ci} \alpha^{32} \mathrm{P}-\mathrm{CTP}(400 \mathrm{Ci} /$ mmol; Amersham, Arlington Heights, IL); $500 \mu \mathrm{M}$ each of GTP, ATP, UTP; $12 \mu \mathrm{M}$ cold CTP; $10 \mathrm{~mm}$ DTT; 20 U of RNasin; and $20 \mathrm{U}$ of T7 polymerase (Promega, Madison, WI) in the buffer supplied by the manufacturer. The reaction was incubated at $37^{\circ} \mathrm{C}$ for $1 \mathrm{hr}$, after which the DNA template was digested with $1 \mathrm{U}$ of RQ1 DNase (Promega) at $37^{\circ} \mathrm{C}$ for $15 \mathrm{~min}$. The transcription reaction was separated by electrophoresis on a $5 \%$ acrylamide/bisacrylamide $8 \mathrm{~m}$ urea gel, and the full-length probe identified by autoradiography then excised and eluted from a small gel slice at $37^{\circ} \mathrm{C}$ in the buffer provided. Each probe $\left(5 \times 10^{4} \mathrm{cpm}\right)$ was hybridized with $30 \mu \mathrm{g}$ of total adult Canton-S RNA in a $20 \mu \mathrm{l}$ reaction volume at $45^{\circ} \mathrm{C}$ overnight. Unprotected RNA was digested by incubation at $37^{\circ} \mathrm{C}$ for $30 \mathrm{~min}$ with a $1: 100$ dilution of the RNase A/RNase TI mixture provided in the kit. RNA was precipitated and separated on a $5 \%$ polyacrylamide $8 \mathrm{M}$ urea gel, in $1 \times$ Tris-borate EDTA buffer. The gel was transferred to chromatography paper and vacuum dried before autoradiography.

\section{Results}

Determining the size and transcript complexity of para

The 1820 amino acids of para cDNA sequence described by Loughney et al. (1989) were incomplete at the carboxyl terminus because the most $3^{\prime}$ cDNA ended in frame without reaching a translation termination codon. The partial sequence of another para cDNA, corresponding to 220 amino acids within homology domain IV, has been reported (Ramaswami and Tanouye, 1989). We obtained this CDNA and determined the remaining sequence (GenBank accession number M32078). The cDNA contained 236 further in-frame amino acids before a termination codon was reached, putting the complete para protein at between 2091 and 2131 residues, the exact size dependent upon which alternative exons are present. The new sequence is presented and compared with $\mathrm{Na}$ channel proteins from rat and eel in Figure 1. Beyond the last presumed transmembrane segment, S6 of homology domain IV, the level of similarity between the three proteins decreases, but a clear alignment remains visible until all but the final $\sim 80$ residues. This cDNA completes the open reading frame of para, the only Drosophila sodium channel sequence known in its entirety.

To determine the size of the para transcript and also to make a general assessment of the extent to which alternative splicing occurs, we probed a Northern blot of polyA ${ }^{+}$RNA from Drosophila heads with a radiolabeled para cDNA fragment. An autoradiograph from this hybridization is shown in Figure 2. A single, although rather broad, band is present at about $15 \mathrm{~kb}$. As the largest RNA molecular weight standard on the gel was $9.5 \mathrm{~kb}$, the size of the para transcript can only be approximately estimated at this size. Although this represents the size of the major species in adult heads, it is possible that para transcripts of other sizes are present in other tissues or at other times in development, or below the level of detection on this autoradio-

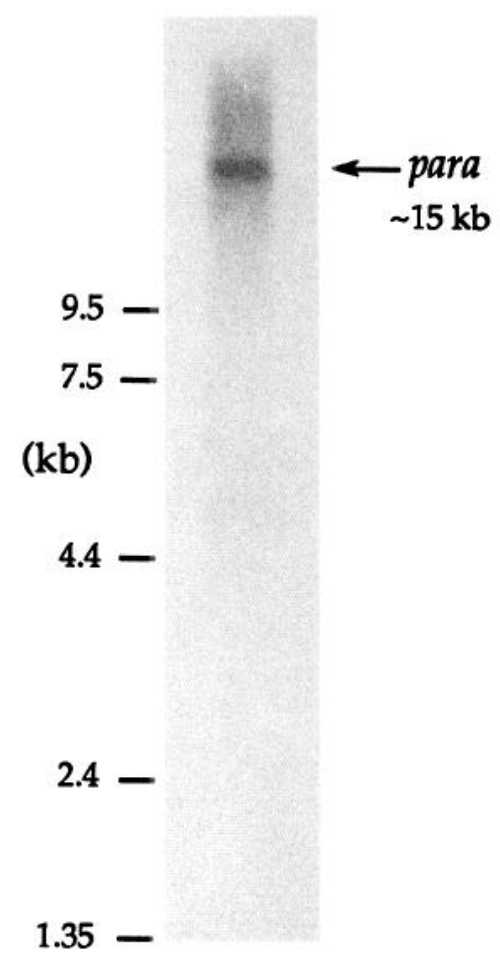

Figure 2. Detection of the para transcript by Northern blotting. A Northern blot of Canton-S, head-specific polyA ${ }^{+}$RNA was probed with a $2.5 \mathrm{~kb}$ PstI/BamHI fragment from para cDNA ZS10.3 (Loughney et al., 1989). The Zetaprobe filter (Bio-Rad) was hybridized according to the manufacturers instructions at $42^{\circ} \mathrm{C}$ overnight. The final stringency of washing was $1 \times$ saline-sodium citrate, $0.1 \%$ SDS; the filter was exposed for autoradiography for $7 \mathrm{~d}$.

graph. However, it is clear that the majority of para mRNAs in adult heads are of a similar size and that alternative splicing leads to only modest variability in the size of para transcripts.

\section{The intron/exon structure of the alternatively spliced region}

To understand the nature of the splicing events used to generate the various para splice forms, we used a combination of Southern blotting, PCR, and sequencing to map the genomic structure of the alternatively spliced region of para within the chromosomal walk described by Loughney at al. (1989). The $1.7 \mathrm{~kb}$ of cDNA sequence that encompasses the known sites of alternative splicing was found to be divided into 13 exons spread over almost $15 \mathrm{~kb}$ of genomic DNA (Fig. 3). Although no intron was found between the exon b sequence and the next $3^{\prime}$ exon (Fig. 3), this juxtaposition of alternatively and constitutively spliced sequences conforms to one of the several known modes of alternative splicing, in which either two donor or two acceptor sites "compete" with each other for a single splice event (Smith et al., 1989). The highly conserved AG dinucleotide that forms part of almost all splice acceptor sequences (e.g., Mount et al., 1992) occurs twice in this region, once within the intron immediately $5^{\prime}$ of the $b$ sequence and a second as the most $3^{\prime}$ pair of nucleotides within exon b itself (Fig. 3). This suggests that para mRNAs either containing or lacking the $\mathrm{b}$ sequence are generated by a selection between these two possible $3^{\prime}$ end points of the preceding intron-either prior to exon b, generating a para product that contains the $\mathrm{b}$ exon, or at the $3^{\prime}$ end of $\mathrm{b}$, resulting in a different product that lacks this exon. A similar situation applies with exon e, in that the e sequence is found at 


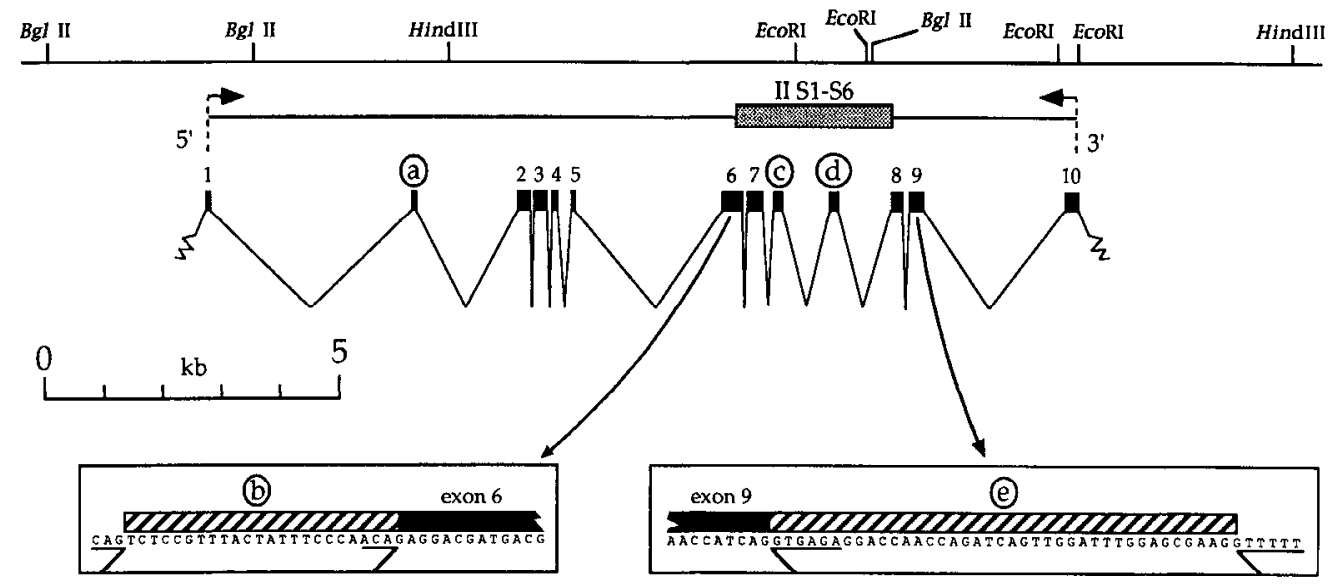

Figure 3. Genomic organization of the alternatively spliced region of para. A restriction map of part of the para genomic region (Loughney et al., 1989) is shown above a schematic representation of the para exon/intron structure in the region under study. Exons are shown as solid boxes, introns as $V$-shaped lines connecting the exons; exons that are alternatively spliced have a circled letter designation. The exons that together encode the second homology domain are indicated by the position of the shaded box between the genomic and exon/intron maps. The primer sites used to amplify para transcripts by RNA-PCR are shown as dashed lines leading to an arrow above the exons to which they hybridize. Exons 6 and 9 are connected directly to alternative exons $b$ and $e$, respectively; the genomic sequences around these two alternative exons are shown in inset beneath the exon/intron structure. Alternatively spliced sequence is shown by hatched boxes; constitutively spliced exon sequence, by solid boxes. The presumed competing splice acceptor sites (Drosophila consensus CAG; Mount et al., 1992) around exon 6 and competing donor sites (Drosophila consensus GTRAGT; Mount et al., 1992) around exon 9 are underlined.

the 3 ' end of exon 9 (Fig. 3). In this instance a selection is presumably made between two possible splice donors in this region, using the GT (the highly conserved dinucleotide found within almost all splice donor sequences; e.g., Mount et al., 1992) either at the $5^{\prime}$ end of exon e to generate para mRNAs that lack exon e, or using the second GT dinucleotide immediately $3^{\prime}$ of exon e to give products that include this exon.

\section{PCR amplification of para transcripts from embryos and adults}

Which of the many possible para splice types are produced in vivo, and when? We used PCR to amplify the $1.7 \mathrm{~kb}$ region of para described above, from total RNA of both embryos and adults, to examine exon usage within intact cDNAs. Although total RNA contains transcripts at all stages of processing, the primer pair chosen for the amplification (within exons 1 and 10; see Fig. 3) are about $15 \mathrm{~kb}$ apart in genomic DNA. We therefore presumed that the vast majority of PCR product obtained with these primers would be from fully spliced para transcripts, due to the amplification length limitations of Taq polymerase. This $1.7 \mathrm{~kb}$ region encompasses about $30 \%$ of the total para coding sequence (between 549 and 593 amino acids of the total 2131) and all the four sites previously known to be alternatively spliced (Loughney et al., 1989). The PCR product was subcloned en masse into a plasmid vector and the many cDNA clones generated analyzed in three restriction enzyme digests to determine which combination of alternative exons was present in each one (Fig. $4 A$ ). In the case of alternative exons $a, b$, and $e$, the digests generated small restriction fragments that encompassed the site of insertion; exons $c, d$, and $f$ were identified by the presence of a unique restriction site within the alternative exon. Although the majority of clones displayed the restriction enzyme digest patterns that could be predicted from the known sequence, some did not, especially those from embryos. Therefore, we also sequenced selected clones, both to determine whether the restriction enzyme analysis gave a true indication of the exons present, and also to explain the origin of the unexpected restriction enzyme patterns.

\section{Sequence analysis of $P C R$-derived $c D N A$ s reveals a new alternative exon}

Twelve clones were sequenced completely, six adult and six embryonic. The clones were chosen such that for each alternative exon site, about as many "exon-present" as "exon-absent" cDNAs would be examined. Aside from occasional single base pair changes presumed to be caused by misincorporation by the Taq polymerase during the PCR amplification, the sequence of each clone agreed with the splice type predicted by the restriction enzyme digests. However, four of the embryonic clones contained a short novel sequence in place of exon e. This exon, now referred to as $\mathrm{f}$, was found in one of two forms, either 24 bp $\left(f^{24}\right)$ or 30 bp $\left(f^{30}\right)$ in length; $f^{24}$ is identical to $f^{30}$, except that 6 bp are missing at the $5^{\prime}$ end (Fig. $4 B$ ). The $f^{30}$ sequence is present in genomic DNA immediately adjacent to exon 10 , without being separated from it by an intron, in the same manner as exon $b$ is to exon 6 (see above). We have not observed a separate sequence corresponding to $\mathrm{f}^{24}$ in genomic DNA; however, there are three potential splice acceptor sequences (AG dinucleotides) present in and around the $\mathrm{f}^{30}$ sequence that could be used to generate all three $f$ variations $\left(\mathrm{f}^{30}, \mathrm{f}^{24}\right.$, or $\left.\mathrm{f}^{-}\right)$from this region of genomic DNA: one at the end of the preceding intron, a second 6 bp from the $5^{\prime}$ end of the fequence, and a third at the $3^{\prime}$ end of the $f$ sequence (Fig. $4 B$ ). Selection of one of these three sites as the $3^{\prime}$ end point of the previous intron could generate any of the three $f$ variants without requiring an additional exon sequence corresponding to $\mathrm{f}^{24}$ to be present elsewhere. The deduced amino acid sequence of exon $f$ does not contain any known consensus sequences for posttranslational modification, and has no significant similarity to any protein domain in the GenBank database.

Four of the 12 fully sequenced clones contained exon c. In each case, the sequence obtained differs from that described by Loughney et al. (1989); the A at position 75 is replaccd by C, resulting in a silent substitution. Because the genomic mapping of exons $c$ did not reveal two copies of this sequence, we conclude that this silent substitution reflects polymorphism within 
or between Canton-S strains rather than the presence of an additional version of exon $\mathrm{c}$.

\section{Eleven embryonic and 18 adult splice types were observed}

To ensure that the splice-type frequencies obtained were not unduly affected by artifactual sources of variation (due to the PCR or the subsequent cloning process, e.g.), we performed independent amplifications from two different preparations of both adult and embryonic RNA. Because of the similarity in the length and sequence of exons $\mathrm{f}^{30}$ and $\mathrm{f}^{24}$, it was difficult to identify which of the two was present in a given clone without sequencing. As a result, we have pooled all the $\mathrm{f}^{30}$ and $\mathrm{f}^{24}$ types simply as $\mathrm{f}^{+}$. While the two amplifications from independent batches of adult RNA gave splice-type distributions that were not significantly different from each other (Monte Carlo simulation; $p=0.14 \pm 0.0011 \mathrm{SE}$; see additional discussion in caption for Fig. $5 A$ ), the two embryonic batches were nonhomogeneous $(p=0.0026 \pm 0.00017 \mathrm{SE})$. Whether the difference between embryonic batches reflects variation between the two different batches of RNA, PCR amplifications, or the subcloning procedure is unclear; however, the homogeneity between the adult batches suggests that the problem of multiple isolation of the same original clone in PCR was not a major one. In the analysis that follows we have pooled the two adult batches, but not those from embryos.

The panel of splice types in adults is clearly very different from that observed in either of the batches from embryos (Fig. $5 A$ ). First, the range of splice types is greater in adults than embryos, being composed of 18 and 11 splice types, respectively. It is also interesting to note that the range of splice types observed in the two different stages of development does not completely overlap; there are three embryonic splice types not found in adults and 10 adult types not found in embryos. Second, a striking difference exists between the developmental stages in the distribution of splice types recovered. In embryos, 7 of the 11 splice types are represented by only one or two clones, and there is a heavy bias toward two types in particular: fully $75 \%$ (100 of 133 ) of the embryonic clones $(69 \%$ and $78 \%$ in the two batches) lack exons $\mathrm{c}$ and e but contain exons $\mathrm{b}, \mathrm{d}$, and $\mathrm{f}$, exon a being present in one group but not in the other (given as $\left[\mathrm{a}^{+}\right.$ or $\left.\mathrm{a}^{-} ; \mathrm{b}^{+} ; \mathrm{d} ; \mathrm{e}^{-} ; \mathrm{f}^{+}\right]$). By contrast, the adult clones are much more evenly spread between the different splice types - the two most common adult types $\left(\left[\mathrm{a}^{+}\right.\right.$or $\left.\left.\mathrm{a}^{-} ; \mathrm{b}^{+} ; \mathrm{d} ; \mathrm{e}^{-} ; \mathrm{f}^{-}\right]\right)$represent only $40 \%$ of the total.

\section{The usage of individual exons changes during development}

Individual exon usage is compared between embryos and adults in Figure $5 B$. In keeping with the narrower range of splice types observed in embryos, there is much greater uniformity in individual alternative exon choice. The exons used at the $\mathrm{c} / \mathrm{d}$, e, and $\mathrm{f}$ sites are almost completely invariant in embryonic para transcripts, being [d, $\mathrm{e}^{-}, \mathrm{f}^{+}$] in $87 \%$ of all clones, with only 2 of 133 clones containing exon $c$ and only 4 of 133 exon e. Although the splicing of exons $\mathrm{a}$ and $\mathrm{b}$ is more variable, even at those sites $59 \%$ are $\left[\mathrm{a}^{+}, \mathrm{b}^{+}\right]$. A much more variable pattern of splicing is evident in the adult clones. Usage of exon a drops to about half of all clones $(51.3 \%$ compared to $80.6 \%$ and $62.3 \%$ in the two embryonic batches), exon b usage drops slightly to $78.2 \%$ (from $92.5 \%$ and $84.9 \%$ in embryos), exon c is used in about a quarter of all clones ( $24.3 \%$ compared to $1.5 \%$ and $1.3 \%$ ), and exon e usage increases to $27.6 \%$ ( $1.5 \%$ and $4.2 \%)$. However, the most dramatic change occurs for exon $f$, which switches from
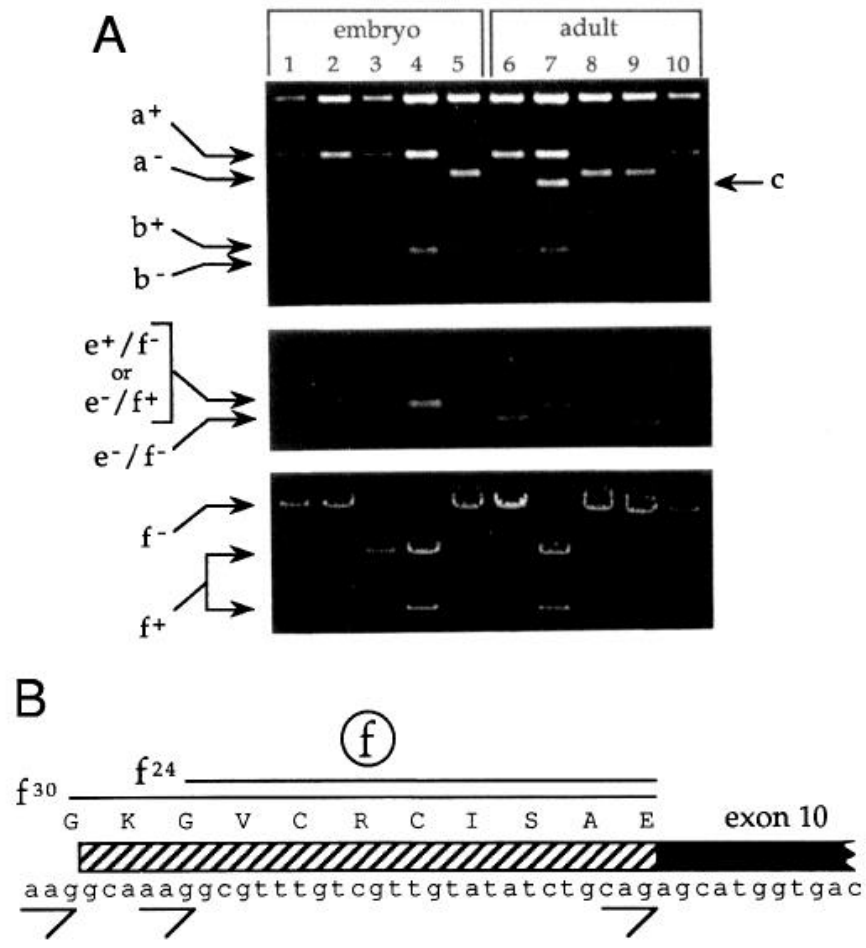

Figure 4. Characterization of PCR-derived para cDNAs and identification of a new alternative exon. $A$, Example of the restriction enzyme digests used to determine the alternative exons present in each para cDNA. Three ethidium bromide-stained agarose gels are shown, containing digests for each of 10 independent RNA-PCR-derived para cDNAs. Lanes 1-5 are clones derived from embryonic RNA; lanes 610 are derived from adult RNA. The enzymes used in each case were ApaLI/ClaI (top), StuI/XbaI (middle), and PstI/HindIII (bottom). Restriction fragments used to determine which alternative exons were present in each cDNA are indicated by arrows. The ApaLI/ClaI digest gives either a $504 \mathrm{bp}\left(\mathrm{a}^{+}\right)$or $441 \mathrm{bp}\left(\mathrm{a}^{-}\right)$fragment (in cDNAs containing or lacking exon a, respectively); a $242 \mathrm{bp}\left(\mathrm{b}^{+}\right)$or $218 \mathrm{bp}\left(\mathrm{b}^{-}\right)$fragment; or a $1.9 \mathrm{~kb}$ (d) or $1.5 \mathrm{~kb}$ and $416 \mathrm{bp}$ (c) fragment (exon c contains a ClaI site that exon d lacks). The section of the ApaLI/Cla digest (top) containing the $1.9 \mathrm{~kb}$ d-specific band is not shown; where a clone lacked the $\mathrm{c}$-specific band it was designated as containing $\mathrm{d}$ (alternative exons $\mathrm{c}$ and $\mathrm{d}$ are spliced in a mutually exclusive fashion so that each para cDNA always contains either $\mathrm{d}$ or $\mathrm{c}$, but not both). In order to give more direct confirmation that exon $\mathrm{d}$ was present (because the $1.9 \mathrm{~kb}$ d-specific band was sometimes hard to distinguish from other highmolecular-weight bands on the $1.5 \%$ agarose gels used) a PvuII digest was also performed (not shown), which detects an exon d-specific PvuII site that is absent from exon c. The StuI/XbaI digest generates one of six diagnostic fragments: $192 \mathrm{bp}\left(\mathrm{e}^{-}, \mathrm{f}^{-}\right.$clone $), 216 \mathrm{bp}\left(\mathrm{e}^{-}, \mathrm{f}^{24}\right), 222 \mathrm{bp}$ $\left(\mathrm{e}^{-}, \mathrm{f}^{30}\right), 225 \mathrm{bp}\left(\mathrm{e}^{+}, \mathrm{f}^{-}\right), 249 \mathrm{bp}\left(\mathrm{e}^{+}, \mathrm{f}^{24}\right)$, or $255 \mathrm{bp}\left(\mathrm{e}^{+}, \mathrm{f}^{30}\right)$ in length. No $\mathrm{e}^{+}, \mathrm{f}^{+}$clones are shown in the figure due to their rarity; no adult and a total of only two embryonic clones were of this splice type. The HindIII/PstI digest detects a PstI site that occurs in both the 24 and 30 bp versions of exon $\mathrm{f}$; clones lacking exon $\mathrm{f}$ are linearized to a $4.7 \mathrm{~kb}$ fragment, clones containing either $\mathrm{f}^{30}$ or $\mathrm{f}^{24}$ give two fragments of 1.6 and $3.1 \mathrm{~kb}$. As examples, the digests show that clone 4 (embryonic) is splice-type $\left[\mathrm{a}^{+}, \mathrm{b}^{+}, \mathrm{d}, \mathrm{e}^{-}, \mathrm{f}^{+}\right]$; clone 9 (adult) is splice-type $\left[\mathrm{a}^{-}, \mathrm{b}^{-}, \mathrm{d}\right.$, $\left.\mathrm{e}^{-}, \mathrm{f}^{-}\right] . B$, The genomic organization and predicted protein sequence encoded by exon $\mathrm{f}$. The genomic DNA sequence at the $5^{\prime}$ end of exon 10 is shown in lowercase letters; the hatched box indicates exon $\mathrm{f}$ sequence; the solid box indicates sequence at the $5^{\prime}$ end of the constitutively spliced exon 10 . The three possible sites of splicing that generate the different forms of this alternative exon $\left(\mathrm{f}^{30}, \mathrm{f}^{24}\right.$, and $\left.\mathrm{f}^{-}\right)$are indicated by underlining of the postulated acceptor sequences (Drosophila consensus CAG; Mount et al., 1992); horizontal lines also indicate the amino acid sequences encoded by the two forms. The predicted amino acid sequence of exon $\mathrm{f}$ is also shown in uppercase above the hatched $b o x$, using the single-letter code; the $\mathrm{f}^{24}$ form lacks the Gly Lys (GK) pair of amino acids found in $\mathrm{f}^{30}$ splice forms encoded by the $6 \mathrm{bp}$ at the $5^{\prime}$ end of the $f$ sequence. 


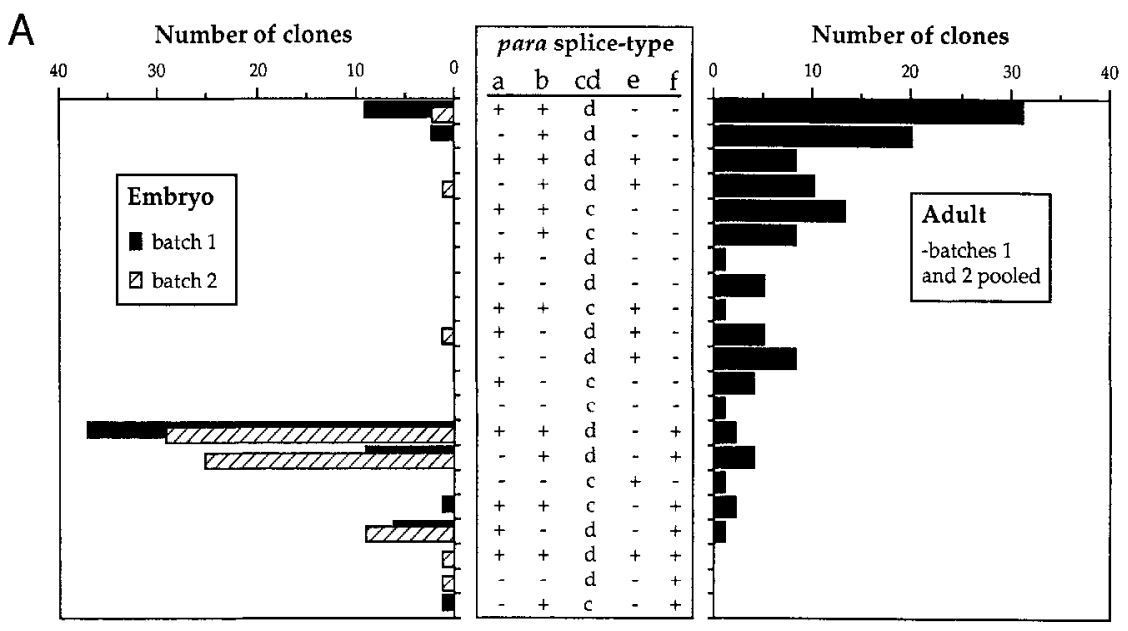

Figure 5. Comparison of para splice types and individual exon usage in embryos and adults. $A$, para splice-type usage in embryos (left) and adults (right). A plus $(+)$ or minus $(-)$ symbol indicates whether a given splice type contains or lacks that exon. A computer program utilizing a Monte Carlo simulation of a row $x$ column contingency table test (W. R. Engels, unpublished) was used to compare the two batches of embryonic clones with each other and the two batches of adult clones with each other. The program uses a method analogous to that described by Lewontin and Felsenstein (1965). Probabilities given in the text were based on simulations of 100,000 trials. $B$, Usage of individual alternative exons in the two embryonic (El and $E 2)$ and combined adult $(A)$ batches. Only those clones in which we have identified the alternative exon at all five positions are included. Each bar gives the percentage of clones containing or lacking the indicated exon. The numbers of $\mathrm{f}^{30}$ and $\mathrm{f}^{24}$ clones are pooled as $f+$ because in most cases we did not determine whether a clone containing $\mathrm{f}$ had the 30 or $24 \mathrm{bp}$ form. $C$, Comparison of observed (solid bars) and expected $(x)$ splice-type frequencies in the combined adult batches. The expected number is based on a model in which exon selection at any site is independent of the alternative exons at other sites.
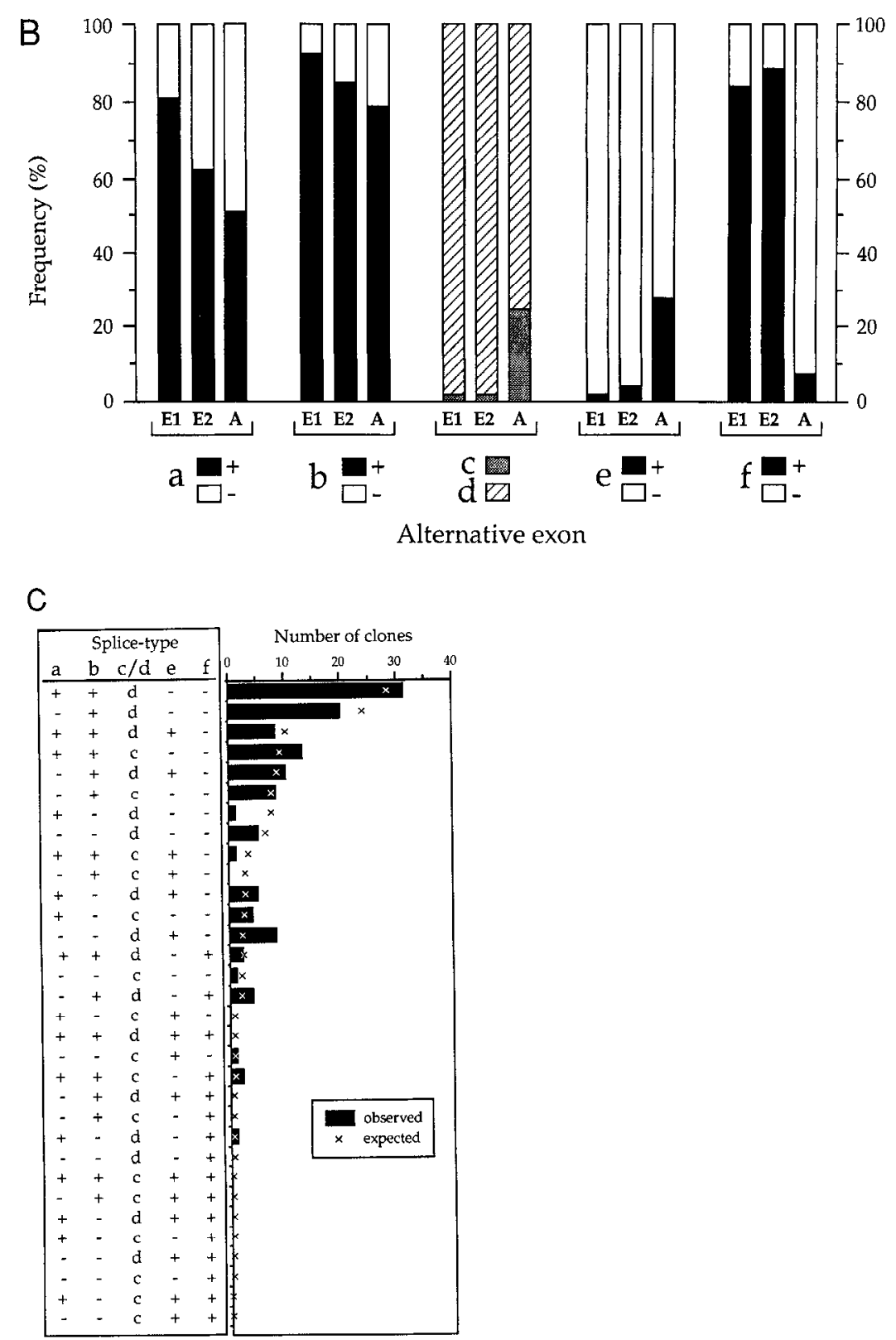
being present in the vast majority of clones in both embryonic batches $(83.6 \%$ and $88.3 \%)$ to a small minority of the adult clones $(6.8 \%)$.

\section{Choices of alternative exons are not independent of each other}

Within each clone, is the presence or absence of a given alternative exon independent of the presence or absence of the other alternative exons? The frequency of any splice type can be predicted, assuming a random splicing choice model, simply by calculating the product of the frequencies of the component alternative exons for all possible splice types. Figure $5 C \mathrm{com}-$ pares the expected frequency of each splice type with the observed frequencies within the pooled adult data given in Figure $5 B$. Although the fit between the observed and expected frequencies appears to be quite good for many of the splice types, they are significantly different overall (goodness of fit $\chi^{2}=35.13$, $\mathrm{df}=16, p<0.005)$. Over half the deviation is accounted for by two splice types in particular; a deficit of splice type $\left[\mathrm{a}^{+}, \mathrm{b}^{-}\right.$, $\mathrm{d}, \mathrm{e}^{-}, \mathrm{f}-\mathrm{]}$ (7.3 expected, 1 observed) and an excess of $\left[\mathrm{a}^{-}, \mathrm{b}^{-}\right.$, $\left.\mathrm{d}, \mathrm{e}^{+}, \mathrm{f}^{-}\right]$(2.2 expected, 8 observed). This suggests that certain pairs (or larger groupings) of alternative exons are spliced into the same mRNA in a nonindependent fashion.

Because we are examining the splice-type frequencies after PCR, it is possible that "selection" for or against particular exons or groups of exons has disturbed these frequencies somewhat. To address this point directly and to be more confident that the data presented above are representative of the situation in vivo, we reexamined the abundance of para alternative exons by a method that does not require an amplification step.

RNase protection assay gives exon frequencies similar to those seen in PCR

An important caveat to the data presented above is that, due to the likelihood of amplification efficiency differences between competitor templates and the exponential nature of the process, PCR is inherently liable to alter the relative frequencies of multiple target sequences during the amplification. The most likely cause of differential amplification efficiency is relative template length, but sequence could also have an effect. To control for any such PCR-induced alteration in relative splice-type frequency, we used an RNase protection assay to compare the abundance of para splice products present in the original RNA pool with those after amplification.

Figure $6 \mathrm{~A}$ shows an autoradiograph of an RNase protection gel, revealing the relative abundance of the alternative exons in adult total RNA, at four of the five sites where alternative splicing occurs. A radiometric scan of the gel was used to determine directly the level of radioactivity within each of the protected bands; the deduced pattern of splicing at each of the four positions examined is compared to that observed in the PCR subclone experiments in Figure $6 B$. Although the inferred exon frequencies from RNase assay are in perfect agreement with PCR in identifying the most frequent splice event at each site (frequency of $\mathrm{a}^{+} \approx \mathrm{a}^{-} ; \mathrm{b}^{+}>\mathrm{b}^{-} ; \mathrm{c}<\mathrm{d} ; \mathrm{e}^{+}<\mathrm{e}^{-}$), the frequencies just reach a significant difference from each other $\left(\chi^{2}=9.29, p\right.$ $<0.05$ ). Although this is most likely to be due to small differences in amplification efficiency, there are other possible explanations for the discrepancies observed between the two assays. Almost half the deviation (4.38 of the total $\chi^{2}$ of 9.29) is due to exon $b$, in which the number of counts in the fully protected band was probably underestimated owing to the need to avoid an adjacent band of undigested probe (marked by a circle in

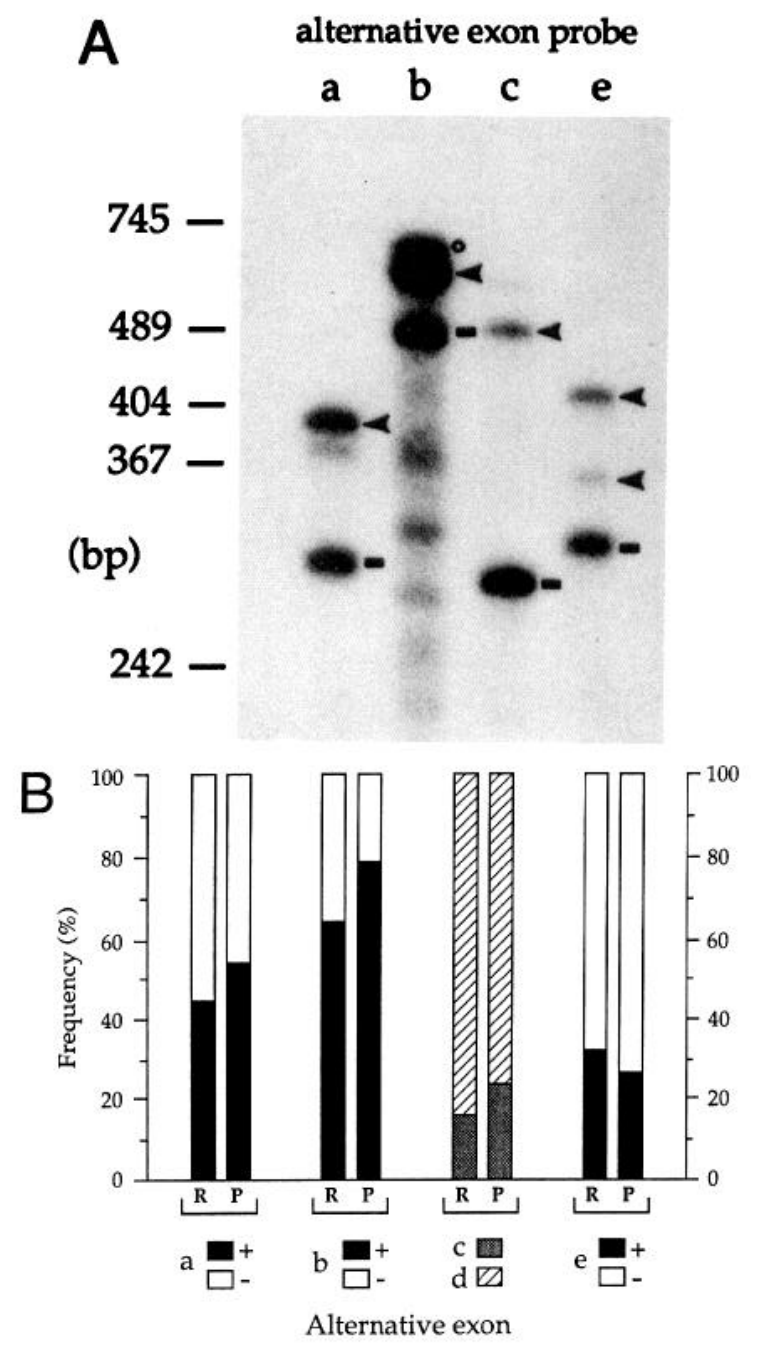

Figure 6. RNase protection of total RNA with probes specific for four of the para alternative exons. $A$, RNase protection assay to determine alternative exon usage in total RNA. Total RNA from Canton-S adults was hybridized with one of four different ${ }^{32} \mathrm{P}$-labeled antisense RNA probes, digested with RNase, and separated on a polyacrylamide gel. Each probe was a para cDNA restriction fragment containing one of the alternative exons. The gel was scanned with a Betascope 603 Blot Scanner (Betagen) for $15 \mathrm{hr}$ and the level of radioactivity within each distinct band determined. A $12 \mathrm{hr}$ autoradiographic exposure of the dried gel is shown here. The fully protected (and therefore exon-present) fragment in each lane is marked with an arrowhead; the cleaved product (generated when the alternative exon was absent) in each lane is marked with a dash (-). Lane e contains two different cleaved fragments because transcripts containing exon e are cleaved if alternative exon $f$ is also present. The substantial band of undigested probe in lane $b$ is marked with a circle. The mean cpm from three apparently blank regions within each lane was used to establish a background signal for each probe and subtracted from the cpm recorded for each protected band. The sizes of protected fragments in base pairs (and cpm recorded) were, for lane $a, \mathrm{a}^{+}=388(14,753), \mathrm{a}^{-}=301(15,059) ;$ lane $b, \mathrm{~b}^{+}=585(40,411), \mathrm{b}^{-}$ $=492(19,195) ;$ lane $c, \mathrm{c}=492(8441), \mathrm{d}=284(24,863)$; lane $e, \mathrm{e}^{+} /$ $\mathrm{f}^{-}=409(7992), \mathrm{e}^{+} / \mathrm{f}^{+}(2989), \mathrm{e}^{-}, \mathrm{f}^{-}=300(15,827) . B$, Bar graph comparing alternative exon usage deduced from PCR and RNase protection. Each pair of bars represents the data derived from RNase protection assay $(R)$ and the RNA-PCR analysis $(P)$. The PCR figures are the adult data presented in Figure $5 B$, in which 68 of the total 125 clones examined contained exon a, 99 exon b, 95 exon $d$ (and therefore 30 exon c), and 33 exon e. The RNase data are derived from the numbers given in $A$, corrected for the number of $\mathrm{G}$ nucleotides in each protected fragment (because CTP was the labeled nucleotide in the antisense probe); the corrected counts (counts/G) were $\mathrm{a}^{+}, 158.6 ; \mathrm{a}^{-}, 195.0 ; \mathrm{b}^{+}, 273.0$; $b^{-}, 152.3 ; \mathrm{c}, 67.0 ; \mathrm{d}, 345.3 ; \mathrm{e}^{+}, 117.4 ; \mathrm{e}^{-} 243.5$. 
Fig. 6A). This underestimation would bring the RNase figure closer to that observed in PCR. Other possible sources of nonartifactual error that may have contributed to the discrepancy between the results from RNase protection and PCR are: first, within-strain polymorphism in the case of exon $\mathrm{c}$ (the exon $\mathrm{c}$ polymorphism described earlier is most likely to have been between strains and, if so, would not be a problem here), causing some clones to be misclassified as containing exon $\mathrm{d}$; and second, the presence of other (as yet unidentified) alternative exons at low frequency, causing overestimation of the frequency of the "exon-absent" condition in RNase protection, or overestimation of the "exon-present" condition in the restriction enzyme analyses of the PCR-derived subclones. However, because we have sequence information from many clones, the frequency of any further novel alternative exons is likely to be very low. Despite these possible sources of error it is evident from Figure $6 B$ that the alternative exon frequencies obtained by the two unrelated methods are quite similar, disagreeing by no more than about $15 \%$ in any of the four sites examined. We conclude that the relative splice-type frequencies observed in the PCR experiment are close to the actual frequencies in the original pool of unamplified cellular mRNA.

The RNase protection result gives us a measure of the accuracy of the PCR-derived alternative exon frequencies, so we can now look back to the data obtained by PCR and be in a better position to assess its significance. Figure $5 B$ presents a comparison of alternative exon frequencies. The change in alternative exon usage from embryo to adult is clearly much greater than could be ascribed simply to PCR-induced effects for three of the five sites: the increased use of exons $c$ and $e$ and the reduction in use of exon $f$. Although the usage of exons a and $b$ may also have decreased to a lesser degree from embryo to adult, the difference is not great enough to be confident that these are developmental changes in gene expression, rather than simply experimental noise. The homogeneity of the two adult PCR samples and the robustness of the PCR-derived frequencics both suggest that the nonindependence of exon usage, shown in Figure $5 C$, is caused by a splicing mechanism rather than an experimental artifact.

\section{Aberrant splice products were also observed}

The majority of the remaining PCR-derived para cDNAs with aberrant restriction enzyme patterns were also sequenced. Most of these were easily explained either by the loss or gain of restriction enzyme sites due to PCR-induced mutation, or by internal deletions of various lengths that sometimes extended into the plasmid multiple cloning site (data not shown). We presume the deletions to be caused by replication slippage during the PCR process or in $E$. coli during transformation, first because these deletions did not take place at the known intron/exon boundaries, and second because a short direct repeat of 6-8 bp was always present at the start and end points of the "missing" sequence (data not shown).

Two other types of aberrant clone were observed that are more likely to be due to aberrant para expression. The first of these was a $10 \mathrm{bp}$ sequence (CTGAAAAAAA) found in at least three clones, two adult and one embryonic, inserted between exons 6 and 7 . Because this is the site where exon $b$ is normally found, we refer to this sequence as b10. The insertion of b10 causes a frame shift that results in translation termination almost immediately, due to the creation of a stop codon $8 \mathrm{bp}$ into exon 7 . Although we have not fully sequenced the $1.6 \mathrm{~kb}$ intron between exons 5 and 6 to locate a possible genomic source of b10, the fact that the same sequence appears in three independent clones suggests that the same missplicing event, using "cryptic" (normally unused) splice donor and acceptor sequences, occurs repeatedly at a low frequency.

The second aberrant class also seems likely to be a splicing error in three embryonic clones, in which the entire sequence of exon 12 was absent. The loss of this 205 bp exon causes a frame shift that would alter the reading frame from S6 of homology domain II onward, presumably leading to loss of channel function. This exon begins with a splice donor consensus dinucleotide GT and ends with a perfect match to the Drosophila splice acceptor sequence CAG, so this sequence may have been mistaken for an intron by the splicing machinery. Although three different clones contained this same deletion, the exon composition of each is identical (data not shown). Thus, these clones may in fact represent repeated isolation of a single event rather than three independent events.

\section{Discussion}

We have used PCR to amplify a region of the para transcript that contains several sites of alternative splicing. By cloning this product and using sequence and restriction enzyme analysis, we have established that a very diverse collection of para $\mathrm{Na}$ channel species is produced, and that the variety of alternative splice products made is much more complex in adults than in embryos. A new alternatively spliced exon was discovered, exon $f$, which was found to be present in one of two different forms, raising the theoretically possible number of para splice types to 48 . From the combined total of 264 PCR-derived cDNAs for which we have complete data (139 embryonic and 125 adult), 19 of the 48 possible types were recovered. How many of the remaining 29 are actually produced in vivo? The fact that 6 of the 19 types are represented by a single clone suggests that we have not reached saturation coverage of the possible splice types in the experiments described here. By scrcening further PCR-derived para $\mathrm{cDNA}$ libraries with probes corresponding to the less frequently used exons - such as $\mathrm{c}, \mathrm{e}$, or $\mathrm{f}-$ we would predict that further novel combinations of the alternative exons would be recovered.

The actual number of para splice types may well be much larger even than 48 , because the region examined in detail in this study represents only about $30 \%$ of the complete para open reading frame. Approximately one-third of the para sequence is known only from a single cDNA sequence (Loughney et al, 1989), so any alternative splicing in this region would not have been detected; even in those places represented by two overlapping cDNA sequences, a rarely utilized exon would also remain undetected. Indeed, recent analysis of alternative splicing in the para homolog from Drosophila virilis (J. R. Thackeray and B. Ganetzky, unpublished observations) indicates that further alternative exons are present; in addition to the same alternative exons observed in $D$. melanogaster, a new alternatively spliced exon was observed just $3^{\prime}$ of the $1.7 \mathrm{~kb}$ region amplified in $D$. melanogaster. Thus, we may be well short of determining the true level of para's complexity.

Exons 9 and 10, which contain the e and f sequences, respectively (Fig. 3), can be spliced together in six different ways. The splicing machinery can "choose" between two different 5 and three different $3^{\prime}$ ends of this one intron, resulting in one of six different open reading frames (Fig. 7). Why is there such variety in splice products at this position, especially considering 
that the cytoplasmic linking region between homology domains II and III, where the exon $e$ and $f$ sequences reside, is very poorly conserved between vertebrate and invertebrate Na channels? Although this domain has not yet been ascribed a particular function, the high degree of protein sequence flexibility in this region of the para protein suggests that it may modulate channel function in some way. A functional role for exon $f$ is also suggested by the fact that the usage of this exon changes so dramatically between embryos and adults. In addition, its presence in the vast majority of embryonic clones $(83.6 \%$ and $88.3 \%$ in the two batches), compared to a small minority of adult clones $(7 \%)$, suggests that it is involved in a sodium channel function almost exclusive to embryos. $\Lambda$ similar case of developmentally regulated alternative exon use has been described in the rat RII/ RII2A Na channel subtype (Sarao et al., 1991; Yarowsky et al., 1991), although the effect on channel function has yet to be demonstrated. The region of the rat $\mathrm{Na}$ channels examined in detail by Schaller et al. (1992) did not include the area homologous to para exons e and $f$; it will be interesting to see whether alternative splicing of similar exons also occurs in this domain of mammalian $\mathrm{Na}$ channels, and if so, whether their presence is also developmentally regulated.

Alternative exon choices were found to be nonindependent. It could be argued that the alternative exon frequencies observed are simply the by-product of sequence-dependent mechanisms within the splicing machinery; however, the change in the panel of para isoforms expressed during development suggests a more biologically meaningful scenario. This is that although there may be many possible splicing pathways for para, certain clusters of alternative exons are "favored," because it is necessary for them to be present together to serve particular functional requirements. It has become clear that, although the primary function of $\mathrm{Na}$ channels in axons is to generate the rapid rising phase of the action potential, in other membranes they can be involved in pacemaker and subthreshold potentials that affect whether a given cell fires or not (Hille, 1992). In mammals an increasingly large family of voltage-gated $\mathrm{Na}$ channels has been cloned, including at least four different genes in rat (Noda et al., 1986; Kayano et al., 1988; Auld et al., 1988; Trimmer et al., 1989). In Drosophila the only likely $\mathrm{Na}$ channel gene known other than para is represented by an incomplete genomic sequence from the DSCl gene (Salkoff et al., 1987), which was identified by low-stringency cross-hybridization with mammalian $\mathrm{Na}$ channel cDNA probes. It may be, then, that Drosophila generates variety in $\mathrm{Na}$ channel function by using alternative splicing of a few genes, rather than by delegating these functions to many different genes.

One unresolved anomaly was the significantly different range of splice types observed in the two amplifications performed on embryonic RNA. However, because the embryos were unstaged-collected en masse 0-24 hr after hatching-it is likely that the embryos in the two batches were not at identical dcvelopmental stages. If the usage of any alternative exon changes during embryonic development, the panel of para splice types being produced would also be altered. Most of the difference between the two batches was in the usage of exon a; in batch 1 exon a was found in $\sim 81 \%$ of all clones (54 of 67 ), whereas in batch 2 this fell to $\sim 62 \%$ (48 of 77 ), so it may be that there is indeed a change in the usage of this exon during embryonic development. An alternative cause of the variation between embryonic batches may have been an increase in sampling error, due to a smaller para mRNA population size at that stage. There

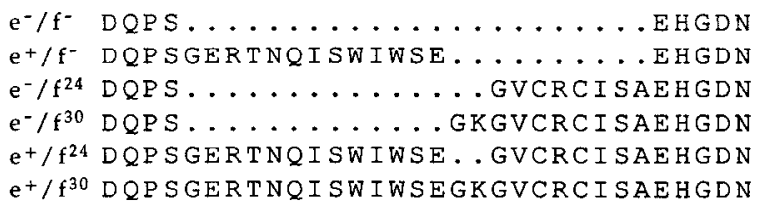

Figure 7. The variety of possible protein sequences encoded by alternative splicing of exons $\mathrm{e}$ and $\mathrm{f}$. The six possible sequences that can be produced by alternative splicing of exons e and $f$ are shown in singleletter code; dots have been introduced to maintain an alignment between the six sequences.

is some evidence to suggest that para is less abundantly expressed in embryos than in adults: the amount of para PCR product from similar reactions was consistently much lower in parallel amplifications from embryonic RNA and adult RNA (data not shown). As a result, multiple PCR reactions from embryos had to be pooled to obtain sufficient material for cloning. This may have resulted in greater stochastic variation in PCR end product, due to the smaller pool of para mRNA in embryos.

\section{Conclusions}

The major finding of this study is that a large family of para $\mathrm{Na}$ channel isoforms are generated by alternative splicing and that the range of isoforms produced changes during development. However, the electrophysiological significance of this variety remains unclear. Because the alternative exon usage alters so dramatically during development, it seems likely that this reflects differing signal transmission requirements in the less complex nervous system of an embryo compared to that of an adult. Exon $f$ shows the most extreme switch, going from a frequency of $87 \%$ in embryos down to only $6.7 \%$ in adults. Exons $c$ and e show lesser but still substantial switches in the opposite direction, going from almost complete absence in embryos ( $1.7 \%$ and $3.4 \%$, respectively) to moderate frequencies in adults $(24 \%$ and $28 \%$ of clones, respectively). Because we do not yet know the mode of para expression within individual neurons - whether a given cell expresses one splice type or many - the basis for these changes in usage may be due either to changes in the abundance of different neuron types or to alteration in the range of splice types expressed within all neurons, or to a mixture of both possibilities. Another reason for believing that the alternative exons may play an important role in para function is that they have been absolutely conserved in position and sequence in the $D$. virilis para homolog (Thackeray and Ganetzky, unpublished observations), after about 60 million years of independent evolution (Beverley and Wilson, 1984). We intend to address the question of alternative exon importance directly, by electrophysiological recording and PCR amplification from single cultured neurons. This method has recently been used to study the rclative cxpression of isoform types from a glutamate receptor (Lambolez et al., 1992). In this way it may be possible to determine whether any differences in $\mathrm{Na}^{+}$currents recorded from different cells are correlated with an alteration in the panel of para splice types being expressed.

\section{References}

Auld VJ, Goldin AL, Krafte DS, Marshall J, Dunn JM, Catterall WA Lester HA, Davidson N, Dunn RJ (1988) A rat brain $\mathrm{Na}^{+}$channel $\alpha$ subunit with novel gating propertics. Ncuron 1:449-461.

Beverley SM, Wilson AC (1984) Molecular evolution in Drosophila 
and the higher Diptera. II. A time scale for fly evolution. J Mol Evol 21:1-13.

Cannon SC, Strittmatter SM (1993) Functional expression of sodium channel mutations identified in families with periodic paralysis. Neuron 10:317-326.

Dascal N, Lotan I (1991) Activation of protein kinase C alters voltage dependence of an $\mathrm{Na}^{+}$channel. Neuron 6:165-175.

Devereux J, Haeberli P, Smithies O (1984) A comprehensive set of sequence-analysis programs for the VAX. Nucleic Acids Res 12:387395.

Drysdale R, Warmke J, Kreber R, Ganetzky B (1991) Molecular characterization of eag: a gene affecting potassium channels in Drosophila melanogaster. Genetics 127:497-505.

Guy HR, Conti F (1990) Pursuing the structure and function of voltage-gated channels. Trends Neurosci 13:201-206.

Hille B (1992) Ionic channels of excitable membranes, $2 \mathrm{~d}$ ed. Sunderland, MA: Sinauer.

Hodgkin AL, Huxley AF (1952) A quantitative description of membrane current and its application to conduction and excitation in nerve. J Physiol (Lond) 117:500-544.

Kayano T, Noda M, Flockerzi V, Takahashi H, Numa S (1988) Primary structure of rat brain sodium channel III deduced from the cDNA sequence. FEBS Lett 228:187-194.

Lambolez B, Audinat E, Bochet P, Crépel F, Rossier J (1992) AMPA receptor subunits expressed by single Purkinje cells. Neuron 9:247258.

Lewontin RC, Felsenstein J (1965) Robustness of homogeneity tests in 2xN tables. Biometrics 21:19-33.

Li M, West JW, Lai Y, Scheuer T, Catterall WA (1992) Functional modulation of brain sodium channels by cAMP-dependent phosphorylation. Neuron 8:1151-1159.

Loughney K, Kreber R, Ganetzky B (1989) Molecular analysis of the para locus, a sodium channel gene in Drosophila. Cell 58:1143-1154.

Mount SM, Burks C, Hertz G, Stormo GD, White O, Fields C (1992) Splicing signals in Drosophila: intron size, information content and consensus sequences. Nucleic Acids Res 20:4255-4262.

Noda M, Shimizu S, Tanabe T, Takai T, Kayano T, Ikeda T, Takahashi $H$, Nakayama $H$, Kanaoka Y, Minamino N, Kangawa K, Matsuo M, Raftery A, Hirose T, Inayama S, Hayashida H, Miyata T, Numa S (1984) Primary structure of Electrophorus electricus sodium channel deduced from cDNA sequence. Nature 312:121-127.

Noda M, Ikeda T, Kayano T, Suzuki H, Takeshima H, Kurasaki M, Takahashi H, Numa S (1986) Existence of distinct sodium channel messenger RNAs in rat brain. Nature 320:188-192.

Perney TM, Kaczmarek LK (1991) The molecular biology of $\mathrm{K}^{+}$channels. Curr Opin Cell Biol 3:663-670.

Ptácek LJ, George AL Jr, Griggs RC, Tawil R, Kallen RG, Barchi RL, Robertson M, Leppert MF (1991) Identification of a mutation in the gene causing hyperkalemic periodic paralysis. Cell 67:1021-1027.
Ramaswami M, Tanouye M (1989) Two sodium channels in Drosophila: implications for channel diversity. Proc Natl Acad Sci USA 86:2079-2082.

Rossie S, Catterall WA (1989) Phosphorylation of the alpha-subunit of rat brain sodium channels by cAMP-dependent protein kinase at a new site containing Ser686 and Ser687. J Biol Chem 264:1422014224.

Rossie S, Gordon D, Catterall WA (1987) Identification of an intracellular domain of the sodium channel having multiple cAMP-dependent phosphorylation sites. J Biol Chem 262:17530-17535.

Salkoff L, Butler A, Wei A, Scavarda N, Giffen K, Ifune C, Goodman R, Mandel G (1987) Genomic organization and deduced amino acid sequence of a putative sodium channel gene in Drosophila. Science 237:744-749.

Sambrook J, Fritsch EF, Maniatis T (1989) Molecular cloning, a laboratory manual, $2 \mathrm{~d}$ ed. New York, NY: Cold Spring Harbor Laboratory.

Sarao R, Gupta SK, Auld VJ, Dunn RJ (1991) Developmentally regulated alternative RNA splicing of rat brain sodium channel mRNAs. Nucleic Acids Res 19:5673-5679.

Schaller KL, Krzemien DM, McKenna NM, Caldwell JH (1992) Alternatively spliced sodium channel transcripts in brain and muscle. J Neurosci 12:1370-1381.

Schwarz T, Papazian D, Carretto R, Jan Y, Jan I, (1990) Immunological characterization of $\mathrm{K}^{+}$channel components from the Shaker locus and differential distribution of splicing variants in Drosophila. Neuron 2:119-127.

Siddiqi O, Benzer S (1976) Neurophysiological defects in temperaturesensitive mutants of Drosophila melanogaster. Proc Natl Acad Sci USA 73:3253-3257.

Sigel E, Baur R (1988) Activation of protein kinase C differentially modulates neuronal $\mathrm{Na}^{+}, \mathrm{Ca}^{++}$and $\gamma$ aminobutyrate type-A channels. Proc Natl Acad Sci USA 85:6192-6196.

Smith CWJ, Patton JG, Nadal-Ginard B (1989) Alternative splicing in the control of gene expression. Annu Rev Genet 23:527-577.

Suzuki DT, Grigliatti T, Williamson R (1971) Temperature-sensitive mutations in Drosophila melanogaster, VII. A mutation (parass) causing reversible adult paralysis. Proc Natl Acad Sci USA 68:890-893.

Trimmer JS, Cooperman SS, Tomiko SA, Zhou J, Crean SM, Boyle MB, Kallen RG, Sheng Z, Barchi RL, Sigworth FJ, Goodman RH, Agnew WS, Mandel G (1989) Primary structure and functional expression of a mammalian skeletal muscle sodium channel. Neuron $3: 33-49$.

Wu C-F, Ganetzky B (1980) Genetic alteration of nerve membrane excitability in temperature-sensitive paralytic mutants of Drosophila melanogaster. Nature 286:814-816.

Yarowsky PJ, Krueger BK, Olson CE, Clevinger EC, Koos RD (1991) Brain and heart sodium channel subtype mRNA expression in rat and cerebral cortex. Proc Natl Acad Sci USA 88:9453-9457. 